\title{
PAPER
}

\section{Sex differences in past tense overregularization}

\section{Evan Kidd ${ }^{1}$ and Jarrad A.G. Lum ${ }^{2}$}

1. School of Psychological Sciences, The University of Manchester, UK

2. School of Psychology, Deakin University, Australia

\begin{abstract}
Hartshorne and Ullman (2006) presented naturalistic language data from 25 children (15 boys, 10 girls) and showed that girls produced more past tense overregularization errors than did boys. In particular, girls were more likely to overregularize irregular verbs whose stems share phonological similarities with regular verbs. It was argued that the result supported the Declarativel Procedural model of language, a neuropsychological analogue of the dual-route approach to language. In the current study we present experimental data that are inconsistent with these naturalistic data. Eighty children (40 males, 40 females) aged 5;0 6;9 completed a past tense elicitation task, a test of declarative memory, and a test of non-verbal intelligence. The results revealed no sex differences on any of the measures. Instead, the best predictors of overregularization rates were item-level features of the test verbs. We discuss the results within the context of dual versus single route debate on past tense acquisition.
\end{abstract}

\section{Introduction}

The acquisition and processing of the past tense of verbs has received considerable attention in the psycholinguistic literature because it has the potential to provide insights into one of the more controversial debates in cognitive science: symbolic, rule-based versus distributed or analogybased cognition. In English, the regular past tense is formed via the suffixation of the regular past tense morpheme -ed onto the base form of the verb (e.g. walkwalked). In contrast, the past form of irregular verbs is less predictable, and in English requires an internal change to the stem itself (e.g. bring-brought, ring-rang) or zero marking (e.g. hit-hit). Dual route approaches (e.g. Marcus, Pinker, Ullman, Hollander, Rosen \& Xu, 1992; Pinker, 1999; Ullman, 2001) argue that regular and irregular verbs are processed via two separate mechanisms: the past tense of regular verbs is formed via a symbolic rule ('add - ed to a regular verb stem'), whereas the past forms of irregular verbs must be memorized and stored as separate entries in the lexicon. In contrast, single route approaches argue that a single associative learning mechanism is responsible for the formation of all past tense forms; morphological knowledge is argued to be an emergent property of analogies made over features of verbs stored in the lexicon (e.g. Bybee, 1995; Joanisse \& Seidenberg, 1999; Rumelhart \& McClelland, 1986).

There have been a host of studies published in the language acquisition literature that argue for or against both approaches. Although many early studies focused on the trajectory of development (e.g. Brown, 1973; Kuczaj, 1977), later studies have attempted to explain children's overregularization (OR) errors (e.g. Marcus et al., 1992; Marchman, 1997; Marchmann, Wulfleck \& Ellis Weismer, 1999; Maratsos, 2000; Maslen, Theakston, Lieven \& Tomasello, 2004; Stemberger, 1993). Most, if not all children will pass through a stage in development where they make OR errors (e.g. goed, singed, hitted). Dual route approaches explain these errors as an overapplication of the regular rule to irregular verb stems whose past form has failed to be retrieved from associative memory. Since the regular rule is argued to be a default (Marcus et al., 1992), the dual route approach predicts that, all things being equal, any verb for which there is not evidence of irregularity will be processed via the symbolic route. A 'blocking mechanism' prevents the application of the rule to any verb for which there is sufficient evidence of irregularity. This is dependent on the frequency of the irregular past form in associative memory: high frequency irregulars are less vulnerable to OR because they will have a stronger memory trace, making lexical access easier. In contrast, the single route approach explains ORs by appealing primarily to type frequency; that is, the regular rule is over-applied because it is by far the most frequent way of forming the past tense (Bybee, 1995).

An important difference between the dual and single route approaches is that the approaches make different

Address for correspondence: Evan Kidd, School of Psychological Sciences, The University of Manchester, Oxford Road, Manchester M13 9PL, UK; e-mail: evan.j.kidd@manchester.ac.uk or Jarrad A.G. Lum, School of Psychology, Deakin University (Waterfront), 1 Gheringhap Street, Geelong 3217, Australia; e-mail: jarrad.lum@deakin.edu.au 
predictions regarding children's OR errors. Although both approaches predict that the frequency of the past form will affect OR rates, only the single route approach indicates that OR errors are predictable from the phonological properties of verbs (Marchman, 1997; Stemberger, 1993). Stemberger (1993) re-analysed Marcus et al.'s (1992) naturalistic data and showed that children's OR errors reflect sensitivity to the phonological features of both verb stems and past forms. Marchman (1997, see also Marchman et al., 1999) reported experimental data from an elicited production task conducted with children aged 3-13 years and showed that their OR errors were predictable from (i) frequency of past form, (ii) stem-final phonology (alveolar or non-alveolar), and (iii) phonological neighbourhood structure. These results provide the most convincing support for the single route approach.

Recently, Hartshorne and Ullman (2006) reported on the OR rates of 25 children whose naturalistic speech was recorded at varying frequencies from age $1 ; 4$ to $5 ; 2$. They identified a sex difference in OR rates: girls made more OR errors than did boys, a result that in their sample could not be attributed to age, socioeconomic status, ethnicity, or parental input, as measured by the number of past tense tokens directed to the children by their parents in their transcripts. The results indicated that gender accounted for $43.6 \%^{1}\left(\mathrm{CI}_{95}: 12.3 \%, 70.1 \%\right)$ of the variance in OR rates. On closer examination of the children's OR errors they identified that, compared to the boys, the girls in their sample tended to overregularize irregular verbs from dense neighbourhoods that contained similar sounding regular verbs (e.g. link-linked, sink-*sinked, mould-moulded, hold-*holded). They interpreted these data to be consistent with Ullman's (2001, 2004) declarative/procedural (DP) model of language, a neuropsychological analogue of the dual route approach to language.

The DP model attempts to link different facets of language processing to functionally and anatomically distinct parts of the brain. The model argues that so-called rule-based facets of language, such as the processing of regular past forms, are supported by procedural memory. The procedural memory system analyses complex stimuli over repeated exposures, and is responsible for the learning and control of complex skills that are not necessarily available to conscious reflection, such as playing the piano, swimming, and driving a car (Gabrieli, 1998). The DP model argues that irregular past forms are supported by declarative memory. Declarative memory is responsible for making rapid associations between arbitrarily related information. It is responsible for lexical learning, where an arbitrary phonological form must be associated with a referent (Eichenbaum \& Cohen, 2001). The memory systems are localized in separate parts of the brain (for extensive reviews see Ullman, 2004; Ullman \& Pierpont, 2005).

${ }^{1}$ Effect size calculated from reported $t$-value and degrees of freedom where $r=\sqrt{t^{2} /\left(t^{2}+d f\right)}$
Hartshorne and Ullman (2006) explained the tendency for girls to produce more OR errors with regular-sounding irregulars by suggesting that because girls have a better declarative memory than boys, they are more likely to store regular past forms in declarative memory, and therefore overregularize similar sounding irregular stems on the basis of analogy. For instance, because hold shares phonological similarities with fold and mould (i.e. they have the same rhyme), girls are more likely to say holded than they are to overregularize forms that do not share phonological similarity with regular verb stems (e.g. $c u t$ ). The suggestion therefore is that there are two ways a child could make OR errors, one via the overapplication of the regular rule, and the other via analogy from similar sounding verbs. The authors claimed that this provided support for the DP model of language.

Our understanding of child language has benefited enormously from the study of language in naturalistic settings (e.g. MacWhinney, 2000). A strength of this approach is that theories tested or derived from such methodologies have a high degree of external validity. However, obtaining data in this manner comes at a cost of being able to examine and/or control variables which may also have influenced or explained the outcome or dependent variable of interest. In the case of Hartshorne and Ullman's (2006) study, no independent tests or assessments of the children's declarative memory abilities were undertaken; they assumed that the 10 girls in their sample had better declarative memory abilities than the 15 boys on the basis of past experimental findings (e.g. Kramer, Delis, Kaplan, O’Donnell \& Prifitera, 1997).

In the first instance it is certainly too strong to assert that females have better declarative memory skills than males. Rather, the evidence indicates subtle differences that vary developmentally. Kramer et al. (1997) tested 401 boys and 410 girls aged from 5 to 16 years on the Californian Verbal Learning Task-Children's Version (CVLT-C; Delis, Freeland, Kramer, Kaplan \& Ober, 1994). On this test children learn a word list over five trials. Children are asked to recall the word lists following each trial and also in short and delayed recall conditions. While Kramer et al. (1997) reported a statistically significant main effect of gender, the magnitude of the effect differed across age groups and recall conditions. Figure 1 shows the effect sizes expressed as $r^{2}$ for gender and age for the fifth learning trial, as well as for the short and delayed recall condition. The value for $r^{2}$ expresses the proportion of variance in the dependent variable (in this case scores from the CVLT-C) that is accounted for by the independent variable, that is, gender. From Figure 1 it can be seen that, at best, gender accounted for only $6.2 \%$ of the variance in the data, ${ }^{2}$ which corresponds to a medium effect size in Cohen's (1988) taxonomy. It is also evident that the effect of gender on declarative memory scores fluctuates over the age groups.

$2 r^{2}$ values computed from means and standard deviations reported in Table 2 of Kramer et al. (1997). 


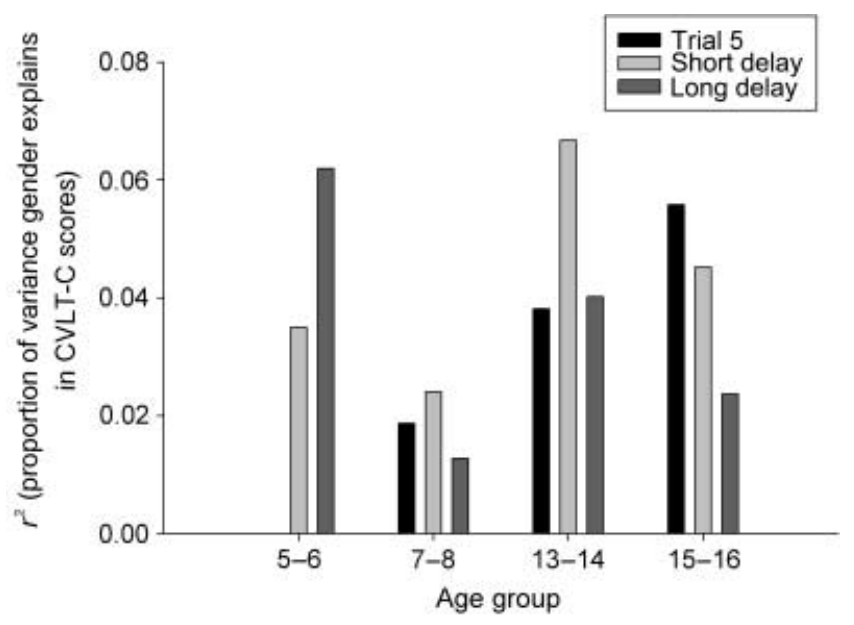

Figure 1 Effect sizes for gender reported by age and recall condition from Kramer et al. (1997). Effect sizes obtained from Ms and SDs reported in Table 2 (Kramer et al., 1997).

\section{Method}

\section{Participants}

A total of 40 female and 40 male children participated in this study. The ages of the female children ranged from $5 ; 0$ to $6 ; 8(M=5 ; 8, S D=0 ; 5)$. The ages of the male children ranged from $5 ; 0$ to $6 ; 9(M=5 ; 9, S D=$ $0 ; 5)$. All children were recruited from schools located in Manchester, UK. The distribution of children's ages was almost identical (Cohen's $d=0.003$ ).

\section{Materials and procedures}

Children completed Marchman, Wulfeck and Ellis Weismer's (1999) Past Tense Task, the Word Pairs subtest from the Children's Memory Scales (WP-CMS; Cohen, 1997), and Raven's Coloured Progressive Matrices (RCPM; Raven. 1998). All tasks were presented individually to each child in a quiet room of their school. The presentation of the tasks was counterbalanced. Each task is described in turn.

\section{Raven's Coloured Progressive Matrices (RCPM)}

The RCPM is a standardized test of nonverbal reasoning. In this test children are presented with a stimulus pattern with a segment missing. Children are asked to identify the missing segment from an array of six possible alternatives. There are a total of 36 items on this test. The male and female children did not significantly differ in their RCPM scores $(t(77)=.433, p=.67$, Cohen's $d$ $=0.10$ ).

\section{Past Tense Elicitation Task}

Children's knowledge of the English past tense was assessed using Marchman et al.'s (1999) Past Tense Task. In this task children are presented with 52 short passages accompanied by stimulus pictures that are designed to elicit past tense forms for regular $(n=25)$ and irregular verbs $(n=27)$. For example, on one item children are presented with a picture of a girl singing. Children are then provided with the stimulus passage 'The girl is singing a pretty song. She sings that song every day. Yesterday she ...' (see Marchman et al., 1999). Children's responses were audio taped and transcribed off-line. In the task, regular and irregular verbs are manipulated on the following dimensions: (i) frequency of the past tense form (high, low), (ii) stem final phonology (alveolar versus non-alveolar), and (iii) phonological neighbourhood properties. In this study we focused on children's performance on irregular verbs with respect to past form frequency (high, low) and whether the irregular verbs sounded similar to a high or low number of regular verbs. We follow Marchman et al. (1999) in calling this final variable 'regular enemies'. The test includes items that have either a high or low number of regular 
enemies; that is, they come from a phonological neighbourhood that has either a high or low number of similar sounding regular verbs.

Children's responses to the past tense task were coded based on the coding scheme used by Marchman et al. (1999).

1. Correct: The child correctly inflects the verb (e.g. walk $\rightarrow$ walked, buy $\rightarrow$ bought).

2. Overregularization: The child affixes the $-e d$ morpheme to an irregular verb (e.g. buy $\rightarrow$ buyed).

3. Zero marked: The child produces the stem in response to the stimulus sentence (e.g. walk $\rightarrow$ walk, buy $\rightarrow$ buy).

4. Invalid: The child responds to the stimulus sentence with the progressive, third person singular, past participle, or responds without using the target verb.

5. Other: All other responses including instances where the child made an inappropriate vowel change to the stem which did not produce the regular past tense form of the verb (e.g. 'yesterday the river flew') or when an incorrect suffix had been added to a regular (e.g. landed $\rightarrow$ landeded).

Only responses to irregular verbs were analysed (see Appendix), since we were interested in children's OR error rates. Following Marchman et al. (1999), the proportion of OR errors was computed for each child as the total number of ORs divided by the sum of the total number of correct and valid responses. This method for measuring ORs differed from Hartshorne and Ullman's (2006) method, but produced very similar scores; the two methods for measuring OR rates were highly correlated $(r=.949, p<.001)$.

Word Pairs from the Children's Memory Scale (WP-CMS; Cohen, 1997)

The WP-CMS is a measure of declarative memory for verbal information. In this subtest children are presented with a list of 10 word pairs (e.g. leaf-school), which they are asked to learn. At the end of each list presentation children are presented with the first word of each pair and prompted for the second. Three trials are presented followed by a further recall trial in which children are asked to recall both pairs. The raw scores on this subtest range from 0 to 40 . The CMS is standardized for children aged between 5 and 16 years. The WP-CMS subtest is standardized to a mean of 10 and standard deviation of 3 .

\section{Results}

\section{Gender differences in declarative memory}

The purpose of the first analysis was to examine whether the female children had superior declarative memory skills for verbal information. The mean standardized scores from the WP-CMS for the female and male children were almost identical (Female: $M=9.0, S D=$ 3.1, Range: 4-17; Male: $M=9.1, S D=3.1$, Range: 5 17). Not surprisingly, an independent samples $t$-test revealed a non-significant difference between the groups $(t(77)=.062, p=.95$, Cohen's $d=0.014)$.

\section{Gender differences in overregularizations}

The next analyses examined gender differences in relation to OR rates. Table 1 presents the means and standard deviations of OR errors for the male and female children by frequency and number of regular enemies. Table 1 shows that males made slightly more OR errors than did females.

These data were analysed using a 2 (Gender: Female, Male) $\times 2$ (Verb Frequency: High, Low $) \times 2$ (Regular Enemies: High, Low) Mixed Design Factorial ANOVA. Overall, there were no differences in OR rates between female and male children $(F(1,77)=.944, p=.334$, partial $\eta^{2}=.012$ ). Furthermore, neither of the interaction terms that included Gender as an independent variable were associated with statistically significant effects (Verb Frequency $\times$ Gender: $F(1,77)=1.070, p=.304$, partial $\eta^{2}=.014$; Regular Enemies $\times$ Gender: $F(1,77)=.177$, $p=.675$, partial $\eta^{2}=.002$; Frequency $\times$ Regular Enemies $\times$ Gender: $F(1,77)=.210, p=.648$, partial $\left.\eta^{2}=.003\right)$.

While gender accounted for only a small percentage of variance in the OR rates, Table 1 shows considerable variability within each group, suggesting individual differences in performance on the past tense task. Similarly, an inspection of the standard deviations associated with

Table 1 Descriptive statistics for male and female children's overregularization rates by verb frequency and number of regular enemies

\begin{tabular}{|c|c|c|c|c|c|c|c|c|}
\hline & \multicolumn{8}{|c|}{ Verb frequency } \\
\hline & \multicolumn{4}{|c|}{ Low } & \multicolumn{4}{|c|}{ High } \\
\hline & \multicolumn{2}{|c|}{ Low regular enemies } & \multicolumn{2}{|c|}{ High regular enemies } & \multicolumn{2}{|c|}{ Low regular enemies } & \multicolumn{2}{|c|}{ High regular enemies } \\
\hline & $M$ & $S D$ & $M$ & $S D$ & $M$ & $S D$ & $M$ & $S D$ \\
\hline Male & .59 & .21 & .60 & .24 & .35 & .25 & .46 & .27 \\
\hline Female & .50 & .26 & .55 & .25 & .33 & .25 & .43 & .27 \\
\hline
\end{tabular}


Table 2 Pearson's r coefficients between WP-CMS (declarative memory) and overregularization rates by gender

\begin{tabular}{lcccrr}
\hline & \multicolumn{2}{c}{ Verb frequency } \\
\cline { 2 - 3 } & \multicolumn{2}{c}{ Low } & & \multicolumn{2}{c}{ High } \\
\cline { 2 - 3 } & Low regular enemies & High regular enemies & & Low regular enemies & High regular enemies \\
\hline Male & $-.161(p=.362)$ & $-.080(p=.652)$ & & $-.032(p=.860)$ & $-.202(p=.253)$ \\
Female & $.176(p=.248)$ & $-.120(p=.433)$ & & $.105(p=.492)$ & $.058(p=.704)$ \\
\hline
\end{tabular}

Table 3 Descriptive statistics for age, non-verbal reasoning and declarative memory by gender

\begin{tabular}{|c|c|c|c|c|c|c|c|c|c|}
\hline & \multicolumn{3}{|c|}{ Age } & \multicolumn{3}{|c|}{ Non-verbal reasoning } & \multicolumn{3}{|c|}{ Declarative memory } \\
\hline & $M$ & $S D$ & Range (min-max) & $M$ & $S D$ & Range (min-max) & $M$ & $S D$ & Range (min-max) \\
\hline Female & 5.82 & 0.51 & $5.08-6.75$ & 103.14 & 12.53 & $75-122$ & 11.9 & 1.65 & $9-17$ \\
\hline Male & 5.68 & 0.41 & $5.00-6.75$ & 102.70 & 17.03 & $75-124$ & 7.1 & 2.15 & $5-9$ \\
\hline$t$-test & \multicolumn{3}{|c|}{$t(38)=.968, p=.339$, Cohen's $d=.30$} & \multicolumn{3}{|c|}{$t(38)=.101, p=.920$, Cohen's $d=.03$} & \multicolumn{3}{|c|}{$t(38)=7.919, p<.001$, Cohen's $d=2.50$} \\
\hline
\end{tabular}

the male and female children's word score pairs also indicates considerable within-group variability. Hartshorne and Ullman (2006) suggested that individual gender differences in declarative memory skills may also explain OR rates. To explore this issue we examined the correlation between WP-CMS scores and OR rates separately for the male and female children. Since the DP model predicts that declarative memory should predict OR errors on irregular verbs that have a high number of regular enemies, we performed eight separate correlations that respected the manipulations in the previous analysis (i.e. Gender $x$ Verb Frequency $\times$ Regular Enemies). The results are presented in Table 2. In all cases the magnitude of the correlation coefficients approached zero; none were significantly different from zero.

Failure to find a meaningful effect of Gender on OR rates in the previous analysis may have arisen because the male and female children did not differ with respect to declarative memory skills. That is, the non-significant difference in WP-CMS scores between female and male children complicated our attempt to test Hartshorne and Ullman's (2006) claim that girls have superior declarative memory abilities, which in turn should lead to more overregularizations of irregulars with a high proportion of regular neighbours. Our comparison suggests that girls do not have better declarative memory abilities than boys at this age. Despite this result, it is not impossible that the female children studied by Hartshorne and Ullman had superior declarative memory skills than the male children. However, as mentioned earlier, since they did not have an independent test of their children's declarative memory abilities, their results must be interpreted with caution. To address this concern, new groups of female and male children were created using standardized scores from the WP-CMS. The female group consisted of 20 children who obtained the highest standardized scores from the WP-CMS from all sampled female children. The male group consisted of 20 children who obtained the lowest standardized scores of sampled males. These two groups were also matched on age and non-verbal intelligence. Summary statistics pertaining to age, WP-CMS, and RCPM scores are presented in Table 3.

Differences between the groups were evaluated using a series of independent samples $t$-tests and Cohen's $d$. As expected, the groups differed only in their declarative memory ability, as measured by their WP-CMS standardized scores. Importantly, it should be noted that there was virtually no overlap in scores between the female and male children with respect to declarative memory. The highest score for the male group was equal to the lowest score for the female group. Devising groups in this manner allowed for a controlled test of Hartshorne and Ullman's (2006) proposal. First, the children were matched on age and non-verbal intelligence. Second, the female children had higher declarative memory skills. Third, children's OR errors were obtained under similar circumstances, since they all received the same elicitation task.

The analyses examined the influence of gender, irregular verb frequency, and regular verb neighbourhood density on children's OR. The mean proportion of ORs by Gender, Verb Frequency and Regular Enemies are presented in Table 4. These data were analysed using a 2 (Gender: Female, Male $) \times 2($ Verb Frequency: High, Low $) \times 2$ (Regular Enemies: High, Low) Mixed Design Factorial ANOVA.

The main effect of Gender on children's OR rates was not statistically significant $(F(1,38)=1.60, p=.21$, partial $\left.\eta^{2}=.040\right)$. Furthermore, non-significant effects were also found for all interaction terms that included Gender in the model (Gender $\times$ Frequency: $F(1,38)=.311, p=$ .58 , partial $\eta^{2}=.008$; Gender $\times$ Regular Enemies: $F(1$, $38=2.19, p=.15$, partial $\eta^{2}=.054 ;$ Gender $\times$ Frequency 
Table 4 Proportion of ORs by gender, verb frequency and number of regular enemies

\begin{tabular}{|c|c|c|c|c|c|c|c|c|}
\hline & \multicolumn{8}{|c|}{ Verb frequency } \\
\hline & \multicolumn{4}{|c|}{ Low } & \multicolumn{4}{|c|}{ High } \\
\hline & \multicolumn{2}{|c|}{ Low regular enemies } & \multicolumn{2}{|c|}{ High regular enemies } & \multicolumn{2}{|c|}{ Low regular enemies } & \multicolumn{2}{|c|}{ High Regular enemies } \\
\hline & $M$ & $S D$ & $M$ & $S D$ & $M$ & $S D$ & $M$ & $S D$ \\
\hline Male & 0.58 & 0.22 & 0.61 & 0.24 & 0.35 & 0.23 & 0.50 & 0.27 \\
\hline Female & 0.51 & 0.28 & 0.49 & 0.28 & 0.32 & 0.25 & 0.39 & 0.27 \\
\hline
\end{tabular}

$\times$ Regular Enemies: $F(1,38)=.042, p=.84$, partial $\eta^{2}=$ $.001)$. Collectively these results do not show that Gender differences in declarative memory are associated with children's OR rates.

Statistically significant main effects were found for Frequency $\left(F(1,38)=28.94, p<.001\right.$, partial $\left.\eta^{2}=.432\right)$ and Regular Enemies $(F(1,38)=7.24, p=.010$, partial $\eta^{2}=.160$ ). Children were significantly more likely to overregularize verbs that had low past form frequency and high numbers of similar sounding regular neighbours. The interaction between Frequency and Number of Regular Enemies fell short of statistical significance $\left(F(1,38)=3.52, p=.07\right.$, partial $\left.\eta^{2}=.085\right)$.

\section{Discussion}

The central findings to emerge from this study were that (i) declarative memory abilities did not differ according to gender in young primary school aged children, and (ii) declarative memory was not related to children's tendency to produce OR errors. We did not find evidence to support the claim for a gender difference in children's ORs, and therefore the results are inconsistent with those reported by Hartshorne and Ullman (2006). In our experimental study, gender accounted for only $4 \%$ $\left(\mathrm{CI}_{95}:<.001 \%, 20.75 \%\right)$ of the variance in children's OR errors, whereas Hartshorne and Ullman found that gender accounted for $43.6 \%$ of the variance in children's ORs in their naturalistic data. It is important to note that our obtained effect size was observed even after selectively sampling from the female and male groups so that the girls had a higher declarative memory score, and after controlling for non-verbal intelligence. This was in contrast to Hartshorne and Ullman, who had no independent test of their children's declarative memory abilities, and who therefore could not rule out the possibility that the difference they observed was due to factors other than declarative memory.

Instead, the results from the current study suggest that, following the results of Marchman et al. (1999), children's OR errors are predictable from item-level features of verbs independent of gender and declarative memory abilities. This supports the single route approach, which suggests that children construct morphological knowledge in the form of schemas that are constructed via a process of analogies made over forms that are related on a number of dimensions (principally, phonology and semantics) (Bybee, 1995; Joanisse \& Seidenberg, 1999). Therefore, in the current study we found that, in addition to low frequency past forms being vulnerable to OR, so too were irregular verbs that have a large number of similar sounding regular neighbours. This is an explicit prediction of the single route model, irrespective of extraneous variables such as declarative memory abilities.

In contrast, Hartshorne and Ullman (2006) predicted that children make only OR errors on these items if they possess superior declarative memory abilities, a claim for which we found no support. This raises the issue of the exact nature of the relationship between language and long-term memory. Ullman's $(2001,2004)$ DP model of language assumes a direct relationship between the two. However, there is reason to believe that long-term memory plays only an indirect role in the acquisition of past tense morphology. The single route approach predicts that morphological knowledge is an emergent property of analogies made over stored items, and that this same process is responsible for the acquisition of the regular and irregular patterns. Proponents of the single route approach have emphasized the importance of children acquiring a critical mass of verb tokens before they can generalize over similar items to form productive schemas (Marchman \& Bates, 1994; Bates \& Goodman, 1997). Therefore the single route approach claims that while declarative memory is important for acquisition of lexical items, it does not necessarily play a direct role in generalizing over these forms. Support for this claim comes from a recent study presented by Kidd and Lum (2007), who showed that although declarative memory predicts vocabulary size in children aged 4-6 years, it does not directly predict performance on the Marchman et al. (1999) past tense task. Instead, the best predictor of children's success on the task was their vocabulary size. This is consistent with other studies that have reported a direct relationship between vocabulary and grammar in acquisition (Dale, Dionne, Ely \& Plomin, 2000; Dionne, Dale, Boivin \& Plomin, 2003; Marchman, Martínez-Sussman \& Dale, 2004; Marchman, Saccumen \& Wulfeck, 2004; Nicoladis, Palmer \& Marentette, 2007).

One potential criticism of our data is that the children that Hartshorne and Ullman (2006) studied were aged 
5;0 and under, whereas our children were slightly older. We do not see this difference as negating any of our arguments. First, we point out that the children in our study were still making a large number of OR errors, which would have enabled us to identify any gender differences, were they to exist. Second, research by Kramer et al. (1997) indicates that declarative memory differences between female and male children are at their greatest at this age. This gender difference was further augmented in the current study by selecting male and female children on the basis of high and low declarative memory scores. Therefore the potential discrepancies arising from age were thoroughly dealt with in this study.

Overall, we found no evidence to suggest that girls make more OR errors than boys because they have superior declarative memory abilities. Instead, we showed that girls and boys were statistically indistinguishable in their OR error rates, even when we compared a subset of girls who had high declarative memory abilities to a subset of boys who had significantly worse declarative memory, and when these two groups were matched for non-verbal IQ. The pattern of errors was consistent with the single route model, which claims that OR errors are predictable from item-level features of verbs independent of memory (Kidd \& Lum, 2007; Marchman et al., 1999).

\section{Appendix}

Table A List of irregular verbs by frequency of past form and frequency of regular neighbours ${ }^{\text {a }}$

\begin{tabular}{lll}
\hline Item & Frequency & Regular enemies of regulars \\
\hline Cut & High & Low \\
Hurt & High & Low \\
Hit & High & Low \\
Bring & High & Low \\
Break & High & Low \\
Take & High & Low \\
Run & High & Low \\
Eat & High & High \\
Bite & High & High \\
Fall & High & High \\
Go & High & High \\
Stick & High & High \\
Tell & High & High \\
Throw & High & High \\
Sit & Low & Low \\
Build & Low & Low \\
Hold & Low & Low \\
Ride & Low & Low \\
Draw & Low & Low \\
Feel & Low & Low \\
Sing & Low & Low \\
Feed & Low & High \\
Send & Low & High \\
Shed & Low & High \\
Drink & Low & High \\
Drive & Low & High \\
Fly & Low & High \\
\hline & & \\
\hline & & Hew
\end{tabular}

${ }^{a}$ Note: The children were tested on all 52 items used by Marchman et al. (1999). For details of the regular verbs see Marchman et al. (1999).

\section{Acknowledgements}

This research was supported by British Academy grant no. SG-42144. We would like to thank Rachael King and Sarah Davis for help in testing the children, and the schools and children who took part.

\section{References}

Bates, E., \& Goodman, J.C. (1997). On the inseparability of grammar and the lexicon: evidence from acquisition, aphasia, and real-time processing. Language and Cognitive Processes, 12, 507-584.

Brown, R. (1973). A first language. Cambridge, MA: Harvard University Press.

Bybee, J. (1995). Regular morphology and the lexicon. Language and Cognitive Processes, 10, 425-455.

Cherry, L., \& Lewis, M. (1978). Differential socialization of girls and boys: implications for sex differences in language development. In N. Waterson \& C. Snow (Eds.), The development of communication (pp. 189-197). New York: Wiley.

Clark-Stewart, K.A. (1973). Interactions between mothers and their young children: characteristics and consequences. Monographs of the Society for Research in Child Development, 38, Serial No. 153.

Cohen, J. (1988). Statistical power analysis for the behavioural sciences. Hillsdale, NJ: Lawrence Erlbaum Associates.

Cohen, M.J. (1997). Children's memory scales. London: The Psychological Corporation.

Dale, P.S., Dionne, D., Ely, T.C., \& Plomin, R. (2000). Lexical and grammatical development: a behavioral genetic perspective. Journal of Child Language, 27, 619-642.

Delis, D.C., Freeland, J., Kramer, J.H., Kaplan, E., \& Ober, B.A. (1994). The California Verbal Learning Test-Children's Version. San Antonio, TX: Psychological Corporation.

Dionne, G., Dale, P.S., Boivin, M., \& Plomin, R. (2003). Genetic evidence for bidirectional effects of early lexical and grammatical development. Child Development, 74, 394-412.

Eichenbaum, H., \& Cohen, N.J. (2001). From conditioning to conscious reflection: Memory systems of the brain. New York: Oxford University Press.

Gabrieli, J.D. (1998). Cognitive neuroscience of human memory. Annual Review of Psychology, 49, 87-115.

Hartshorne, J.K., \& Ullman, M.T. (2006). Why girls say 'holded' more than boys. Developmental Science, 9, 21-32.

Halverson, C.F., \& Waldrop, M.F. (1970). Maternal behavior toward own and other preschool children: the problem of 'ownness'. Child Development, 41, 839-845.

Huttenlocher, J., Haight, W., Bryk, A., Seltzer, M., \& Lyons, T. (1991). Early vocabulary growth: relation to language input and gender. Developmental Psychology, 27, 236-248.

Joanisse, M.F., \& Seidenberg, M.S. (1999). Impairments in verb morphology following brain injury: a connectionist model. Proceedings of the National Academy of Sciences USA, 96, 7592-7597.

Kidd, E., \& Lum, J.A.G. (2007). Investigating the memory systems underlying the acquisition of English past tense morphology. Unpublished manuscript, The University of Manchester.

Kramer, J.H., Delis, D.C., Kaplan, E., O’Donnell, L., \& Prifitera, A. (1997). Developmental sex differences in verbal learning. Neuropsychology, 11, 577-584. 
Kuczaj, S.A. (1977). The acquisition of regular and irregular past tense forms. Journal of Verbal Learning and Verbal Behavior, 16, 589-600.

MacWhinney, B. (2000). The CHILDES project: Tools for analyzing talk (3rd edn., Vol. 2). Mahwah, NJ: Erlbaum.

Maratsos, M. (2000). More overregularisations after all: new data and discussion on Marcus, Pinker, Ullman, Hollander, Rosen, \& Xu. Journal of Child Language, 27, 183-212.

Marchman, V.A. (1997). Children's productivity in the English past tense: the role of frequency, phonology, and neighbourhood structure. Cognitive Science, 21, 283-304.

Marchman, V.A., \& Bates, E. (1994). Continuity in lexical and morphological development: a test of the critical mass hypothesis. Journal of Child Language, 21, 331-366.

Marchman, V.A., Martínez-Sussman, C., \& Dale, P.S. (2004). The language-specific nature of grammatical development: evidence form bilingual language learners. Developmental Science, 7, 212-224.

Marchman, V.A., Saccuman, C., \& Wulfeck, B. (2004). Productive use of the English past tense in children with focal brain injury and specific language impairment. Brain and Language, 88, 202-214.

Marchman, V., Wulfeck, B., \& Ellis Weismer, S. (1999). Productivity of past tense in children with normal language and specific language impairment. Journal of Speech, Language and Hearing Research, 42, 206-219.

Marcus, G.F., Pinker, S., Ullman, M.T., Hollander, M., Rosen, T., \& Xu, F. (1992). Over-regularization in language acquisition. Monographs of the Society for Research in Child Development, 57, Serial No. 228.

Maslen, R., Theakston, A.L., Lieven, E.V.M., \& Tomasello, M. (2004). A dense corpus study of past tense and plural overgeneralization in English. Journal of Speech, Language, and Hearing Research, 47, 1319-1333.

Nicoladis, E., Palmer, A., \& Marentette, P. (2007). The role of type and token frequency in using past tense morphemes correctly. Developmental Science, 10, 237-254.

Pinker, S. (1999). Words and rules: The ingredients of language. New York: Science Masters.

Plante, E., Schmithorst, V.J., Holland, S.K., \& Byars, A.W. (2006). Sex differences in the activation of language cortex during childhood. Neuropsychologia, 44, 1210-1221.

Raven, J. (1998). Raven's Progressive Matrices and Vocabulary Scales. London: The Psychological Corporation.

Rumelhart, D., \& McClelland, J. (1986). On learning the past tense of English verbs. In D.E. Rumelhart \& J.L. McClelland (Eds.), Parallel distributed processing: Explorations in the microstructure of cognition (Vol. 2): Psychological and biological models (pp. 272-326). Cambridge, MA: MIT Press.

Schachter, F.F. (1979). Everyday mother talk to toddlers: Early intervention. San Diego, CA: Academic Press.

Stemberger, J. (1993). Vowel dominance in over-regularizations. Journal of Child Language, 20, 503-521.

Ullman, M.T. (2001). The declarative/procedural model of lexicon and grammar. Journal of Psycholinguistic Research, 30, 37-69.

Ullman, M.T. (2004). Contributions of memory circuits to language: the declarative/procedural model. Cognition, 92, 231-270.

Ullman, M., \& Pierpont, E.L. (2005). Specific language impairment is not specific to language: the procedural deficit hypothesis. Cortex, 41, 399-433.

Received: 9 July 2007

Accepted: 31 October 2007 